\title{
Selective right middle and lower lobar blockade for minimally invasive cardiac surgery: a prospective, single-center, randomized controlled study
}

\author{
Yun Ren ${ }^{1}$, Yi Lyu ${ }^{2}$, Ying Yu ${ }^{1}$, Lin Jin ${ }^{1}$, Yan $\mathrm{Hu}^{1}$, Kefang Guo ${ }^{1}$, Jing Cang ${ }^{1}$ \\ ${ }^{1}$ Department of Anesthesia, Zhongshan Hospital, Fudan University, Shanghai, China; ${ }^{2}$ Department of Anesthesia, Minhang Hospital, Fudan \\ University, Shanghai, China \\ Contributions: (I) Conception and design: Y Ren, K Guo, J Cang; (II) Administrative support: Y Yu, L Jin, Y Hu; (III) Provision of study materials or \\ patients: Y Ren, Y Lyu, Y Hu; (IV) Collection and assembly of data: Y Ren, Y Lyu, Y Yu, L Jin, Y Hu; (V) Data analysis and interpretation: Y Ren, Y \\ Lyu, K Guo; (VI) Manuscript writing: All authors; (VII) Final approval of manuscript: All authors. \\ Correspondence to: Kefang Guo. Department of Anesthesia, Zhongshan Hospital, Fudan University, No. 1609 Xietu Road, Xuhui District, Shanghai, \\ China. Email: dr_guokefang@163.com.
}

\begin{abstract}
Background: Minimally invasive cardiac surgery (MICS) is increasingly performed due to faster recovery time and lower postoperative complications when compared with the traditional open surgery. However, hypoxemia in lung isolation duration after cardiopulmonary bypass (CPB) surgery has been the focus of anesthesiologists' attention. In the present study, we designed a novel lung isolation strategy to improve oxygenation using a bronchial blocker (BB) to isolate the right middle and lower lobes and preserve the ventilated right upper lobe without affecting the surgical field.

Methods: Patients who had undergone right lateral mini-thoracotomy, a MICS, between August 2018 and February 2019, were enrolled in this randomized controlled study. Patients were randomly divided into a modified lung isolation group (group $\mathrm{M}$ ) and a conventional lung isolation group (group C). In group M, BBs were used to block the bronchus intermedius, while left-sided double lumen endotracheal tubes were used in group $\mathrm{C}$ to isolate the right lung. The primary outcome was to determine the number of patients who required an increase in ventilation volume due to hypoxemia during lung isolation after CPB.

Results: Sixty-one patients (30 in group $\mathrm{C}$ and 31 in group $\mathrm{M}$ ) were enrolled. Five patients in group $\mathrm{M}$ were converted to right lung isolation due to poor surgical field exposure. During lung isolation after CPB, the number of patients with hypoxemia was lower in group $M$ than group $C(5 / 31$ vs. 15/30, $\mathrm{P}=0.005)$.
\end{abstract}

Conclusions: The novel modified lung isolation strategy reduced the incidence of hypoxemia after CPB.

Keywords: Minimally invasive cardiac surgery (MICS); lung isolation; one-lung ventilation; hypoxemia; selective lobar blockade (SLB)

Submitted Jan 21, 2020. Accepted for publication Nov 01, 2020.

doi: $10.21037 /$ atm-20-986

View this article at: http://dx.doi.org/10.21037/atm-20-986

\section{Introduction}

Minimally invasive cardiac surgery (MICS) through right lateral mini-thoracotomy may be an alternative to conventional surgery for patients with mitral valve disease (1). Compared with open surgery, MICS has greater advantages, such as fewer blood transfusion and shorter hospital stay (2), and increasingly more cardiac centers are now performing this surgery as a routine procedure (3).

MICS via the right chest typically requires lung isolation for surgical field exposure, which can be achieved by a double-lumen endotracheal tube (DLT) or a single-lumen endotracheal tube with a bronchial blocker (BB). However, hypoxemia is associated with one-lung ventilation (OLV) after cardiopulmonary bypass (CPB) (4). The surgical 
position of MICS patients also increases intrapulmonary shunt due to the smaller ventilation volume by the left lung (5). Moreover, CPB induces hemodilution, lung interstitial edema, and lung ischemia-reperfusion injury, which impact on pulmonary function and are associated with a significant reduction in oxygenation after CPB (6). Interventions of hypoxemia during OLV include recruitment maneuver, positive end-expiratory pressure (PEEP) to the ventilated lung, and continuous positive airway pressure (CPAP) to the remaining lung. In some patients, hypoxemia cannot be reversed by these methods, nor with $\mathrm{FiO}_{2}$ 1.0. Therefore, anesthesiologists often choose double-lung ventilation (DLV) to maintain oxygenation and the surgical procedure is interrupted.

Patients who had previously undergone contralateral lung lobectomy might be at risk of hypoxemia during OLV due to the limited ventilation volume. For these patients, BB's selective lobar blockade (SLB) should be the preferred ventilation strategy (7). Mini-thoracotomy, which is a MICS, is performed in the fourth intercostal space, where the middle and lower lobes of the right lung are located. In the present study, we present a novel modified lung isolation strategy by blocking the right middle and lower lobes and preserving the right upper lobe. We compared this modified lung isolation strategy with conventional OLV by DLT.

We present the following article following the CONSORT reporting checklist (available at http://dx.doi. org/10.21037/atm-20-986).

\section{Methods}

\section{Research population}

The present study was a prospective, randomized controlled trial. Patients were enrolled between August 2018 and February 2019. The study was conducted in accordance with the Declaration of Helsinki (as revised in 2013) and approved by the Ethics Committee of Zhongshan Hospital, Fudan University (approval number: B2016-022R). All patients provided signed informed consent. The study has been registered on the Clinical trials website (registration number: NCT03505242).

\section{Inclusion and exclusion criteria}

The inclusion criteria were as follows: (I) 18-70 years of age; (II) European System for Cardiac Operative Risk Evaluation II 0-5; and (III) scheduled to undergo elective
MICS through right lateral mini-thoracotomy and femoral cannulation for CPB.

The exclusion criteria were as follows: (I) body mass index $>30 \mathrm{~kg} / \mathrm{m}^{2}$; (II) coronary artery disease; (III) chronic obstructive pulmonary disease or asthma; (IV) previous thoracic or cardiac surgery; (V) infectious endocarditis; and (VI) predicted difficult airway.

\section{Patient allocation}

Enrolled patients were randomly divided into 2 groups (computerized random number, https://www.randomizer. org) 1 day before surgery: a conventional lung isolation strategy group (group C) and a modified lung isolation strategy group (group M). DLTs were used for lung isolation in group $\mathrm{C}$, while BBs were used in group $M$ to block the bronchus intermedius for the right middle and lower lobes collapse during lung isolation.

\section{Anesthesia}

Patients were monitored with an electrocardiogram and pulse oximetry; a central vein catheter and radial artery catheter were then cannulated. Sufentanil, propofol, and rocuronium were used for induction, following tracheal intubation with a Macintosh laryngoscope (Truphatek International Ltd., Netanya, Israel). Intraoperative transesophageal echocardiography was used for all patients. Sevoflurane inhalation (propofol infusion during CPB) was adopted to maintain intraoperative amnesia, along with sufentanil and rocuronium for analgesia and muscle relaxation.

\section{Airway management strategy}

\section{Lung isolation method}

In group C, the left main bronchus diameter was measured by preoperative chest computed tomography. The $35 \mathrm{~F}$ left DLT was selected when the diameter $\leq 11 \mathrm{~mm}$, the 37F DLT, was used for 11-12 mm, and the 39F DLT was used for $>12 \mathrm{~mm}$. After intubation, pediatric fiberoptic bronchoscopy (FOB) was applied for appropriate position confirmation. In group $M$, the ID 8-mm tracheal tube was selected for male patients, and the ID 7.5-mm tracheal tube was selected for female patients. After intubation, FOB was applied to measure the tracheal tube's depth to ensure that the tip of the tube was placed $3-4 \mathrm{~cm}$ away from the main carina. The BB's cuff was placed in the bronchus 


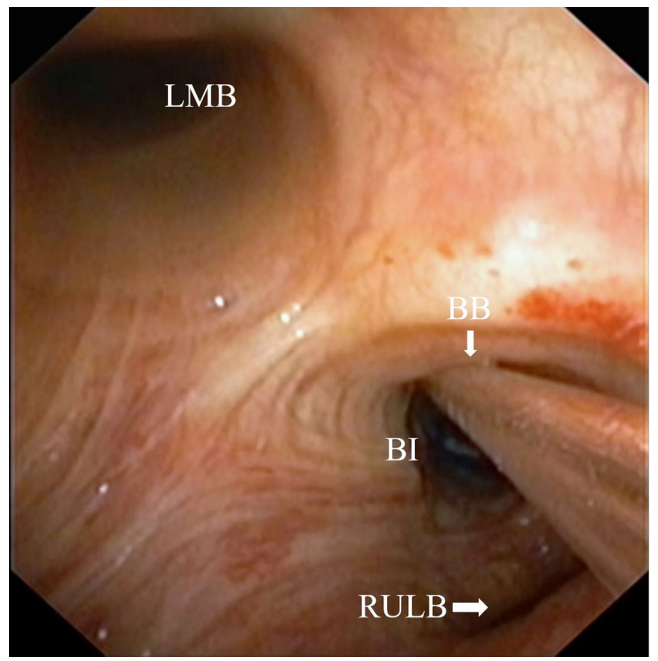

Figure 1 View under fiber optic bronchoscopy. The cuff of BB was placed in the bronchus intermedius with the right middle and lower lobes collapsed and ventilation of the right upper lobe preserved. LMB, left main bronchi; BI, bronchus intermedius; RULB, right upper lobar bronchus; BB, bronchial blocker.

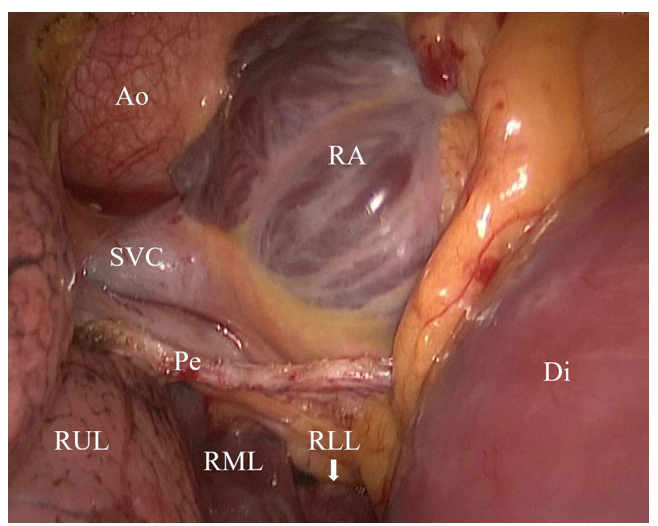

Figure 2 View under thoracoscope. After selective lobar blockade, the right middle and lower lobes collapsed. Ventilation of the right upper lobe was preserved and the surgical field exposure was not affected. Ao, aorta; RA, right atrium; SVC, superior vena cava; Pe, pericardium; Di, diaphragm; RUL, right upper lobe; RML, right middle lobe; RLL, right lower lobe.

intermedius with the guidance of FOB (Figure 1). The cuff position was reconfirmed by auscultation in both groups. All procedures were performed by an attending anesthesiologist who had experience in 10 cases for each method. Also, we recorded the time between the start of the tracheal intubation to the end of lung isolation confirmation.

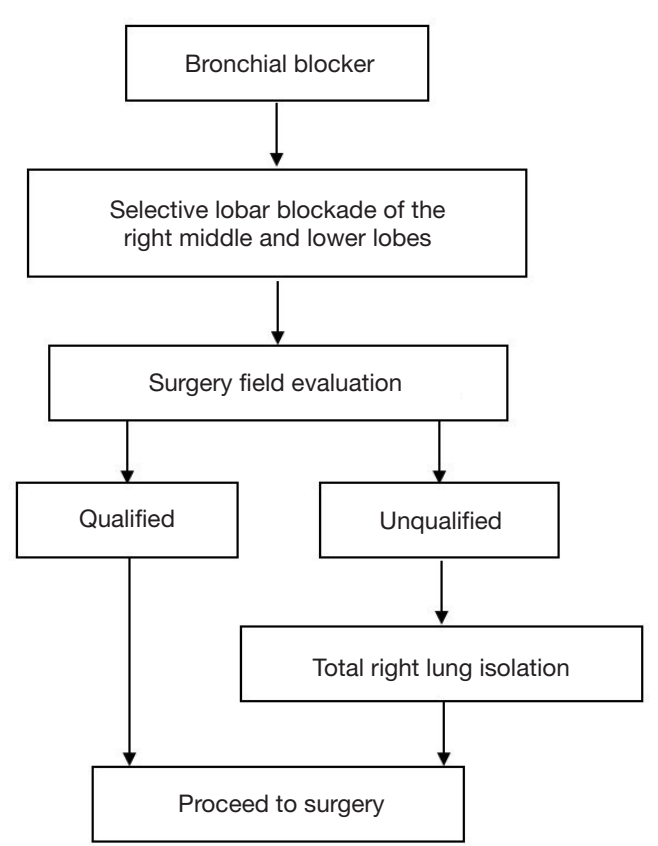

Figure 3 Strategy of modified lung isolation for minimally invasive cardiac surgery.

For pleurectomy to commence, apnea had to last for 1 min in both groups, and the bronchial cuffs in both groups were then inflated to start lung isolation. After the retractor was fixed between the ribs, group M's surgical field exposure was evaluated (Figure 2). If the inflated right upper lobe affected the ascending aorta and aortic root's surgical exposure, mechanical ventilation was suspended for pericardial traction (if hypoxemia occurred during this period, manually controlled DLV with a small tidal volume was applied to assist the pericardial traction). The surgical field exposure was reassessed after restarting mechanical ventilation. If the exposure was still unsatisfactory, the SLB was changed to right lung isolation (Figure 3). Times of intraoperative dislodgement or loss of seal were also recorded.

\section{Intraoperative mechanical ventilation}

After tracheal intubation, volume-controlled mechanical ventilation mode without $\mathrm{PEEP}$ was initiated at $\mathrm{FiO}_{2}$ 1.0, with oxygen flow at $2 \mathrm{~L} / \mathrm{min}$. During DLV, Tidal volume was $8 \mathrm{~mL} / \mathrm{kg}$ (ideal body weight), and the initial respiratory rate was $10 / \mathrm{min}$. Tidal volume during lung isolation was $6 \mathrm{~mL} / \mathrm{kg}$ (ideal body weight), and the initial respiratory rate was $12 / \mathrm{min}$. The inspiratory pause was set to $20 \%$ of the total inspiratory time, and the respiratory rate 
was adjusted to maintain end-tidal $\mathrm{CO}_{2}$ at $40 \pm 5 \mathrm{mmHg}$. Mechanical ventilation was stopped during intracardiac surgical procedures in CPB. Ventilation with lung isolation continued after the aortic unclamping, and the bilateral lung recruitment (pressure $40 \mathrm{cmH}_{2} \mathrm{O}$ for $20 \mathrm{~s}$, the same standard as follows) was performed before CPB weaning. The bloody secretion from the airway suction was defined as airway bleeding. When the surgery was completed, patients in group $\mathrm{C}$ were reintubated with a single-lumen endotracheal tube. At the same time, BB was removed in group $M$. After bilateral lung recruitment, the patients were transferred to the intensive care unit (ICU) for continued respiratory support.

\section{Interventions for hypoxemia}

Hypoxemia was defined as $\mathrm{SpO}_{2}<90 \%$. If hypoxemia occurred during lung isolation, a recruitment maneuver (same standard as described above) was performed to the ventilated lung, and then ventilation under lung isolation continued with $5 \mathrm{cmH}_{2} \mathrm{O}$ PEEP. DLV was implemented if hypoxemia had not resolved. Lung isolation was restarted when $\mathrm{SpO}_{2}>95 \%$, and then $\mathrm{CPAP}$ of $5 \mathrm{cmH}_{2} \mathrm{O}$ was applied to the remaining lung. DLV was directly implemented if hypoxemia occurred in the next lung isolation period.

\section{Surgery and CPB procedure}

The patient was placed in the supine position with a wedge under the right chest. The femoral artery and vein were cannulated to establish CPB. After incision at the fourth intercostal space and pericardium traction, $\mathrm{CPB}$ commenced. The priming solution contained $1,500 \mathrm{~mL}$ Lactated Ringer's injection, $500 \mathrm{~mL}$ Gelofusine, and 100 $\mathrm{mL}$ of $20 \%$ albumin. A target body temperature of $32{ }^{\circ} \mathrm{C}$ and non-pulsating perfusion utilizing a roller pump was adopted.

Furthermore, $\alpha$ state was used for blood gas management. After aortic clamping and antegrade perfusion of blood-containing del Nido cardioplegia (8), surgery was performed through the interatrial groove or the right atrial incision. After rewarming, norepinephrine, dobutamine, and milrinone were transfused to assist weaning from $\mathrm{CPB}$ if necessary. Conventional ultrafiltration was performed during $\mathrm{CPB}$ with a target amount of priming fluid, cardioplegia, and surgical rinsing saline. Residual blood in the surgical field and circuit were collected in an autotransfusion system (Cell Saver Elite; Haemonetics).
After the hemostasis was completed, a chest tube was placed, and the incision was closed.

\section{Data collection}

The respiratory rate, peak airway pressure $\left(\mathrm{P}_{\text {peak }}\right)$, and plateau pressure $\left(\mathrm{P}_{\text {plat }}\right)$ during DLV and lung isolation were recorded in both groups before and after $\mathrm{CPB}$. Blood gas analysis and $\mathrm{PaO}_{2} / \mathrm{FiO}_{2}$ were collected 4 times. $\mathrm{T}_{0}$ indicated data before anesthesia induction $\left(\mathrm{FiO}_{2} 0.21\right), \mathrm{T}_{1}$ indicated data at $10 \mathrm{~min}$ after weaning from CPB (if DLV were performed due to hypoxemia within $10 \mathrm{~min}$, the sample would be collected when the lowest $\mathrm{SpO}_{2}$ occurred in the next lung isolation period), $\mathrm{T}_{2}$ indicated data after bilateral lung recruitment at the end of the surgery, and $\mathrm{T}_{3}$ indicated data $24 \mathrm{~h}$ after surgery. The operation duration, CPB, aortic clamping, and surgical hemostasis (time between weaning from $\mathrm{CPB}$ to the end of surgery), along with volumes, including ultrafiltration, fluid infusion, blood transfusion, and urine, were recorded. The minimum hemoglobin level and the maximum creatinine and cardiac troponin $\mathrm{T}$ levels after surgery were also recorded.

\section{Primary and secondary endpoints}

The primary endpoint was to determine the number of patients who required recruitment maneuvers on the ventilated lung due to hypoxemia during lung isolation after CPB.

The secondary endpoints were to determine the number of patients who required DLV due to hypoxemia during lung isolation after $\mathrm{CPB}$; the time between the start of tracheal intubation to definite confirmation of the position of the lung isolation device, airway bleeding, and dislodgement; the differences in $\mathrm{P}_{\text {peak }}$ and $\mathrm{P}_{\text {plat }}$ during DLV and lung isolation; $\mathrm{PaO}_{2} / \mathrm{FiO}_{2}$ at $\mathrm{T}_{0}, \mathrm{~T}_{1}, \mathrm{~T}_{2}$, and $\mathrm{T}_{3}$ and differences between groups; and evaluation of surgical field exposure [as scored by the surgeon after surgery $(1-5$ points, 1 indicating worst exposure and 5 indicating best exposure)].

\section{Sample size calculation}

Approximately $40 \%$ of patients suffered hypoxemia during lung isolation with DLTs after CPB (unpublished data). However, according to our clinical experience, hypoxemia rarely occurs after $\mathrm{CPB}$ with the successful application of an SLB. Assuming that the modified lung isolation strategy could reduce hypoxemia by $75 \%, 29$ patients were required in each group when simulating the probability of $\alpha=0.05$ 


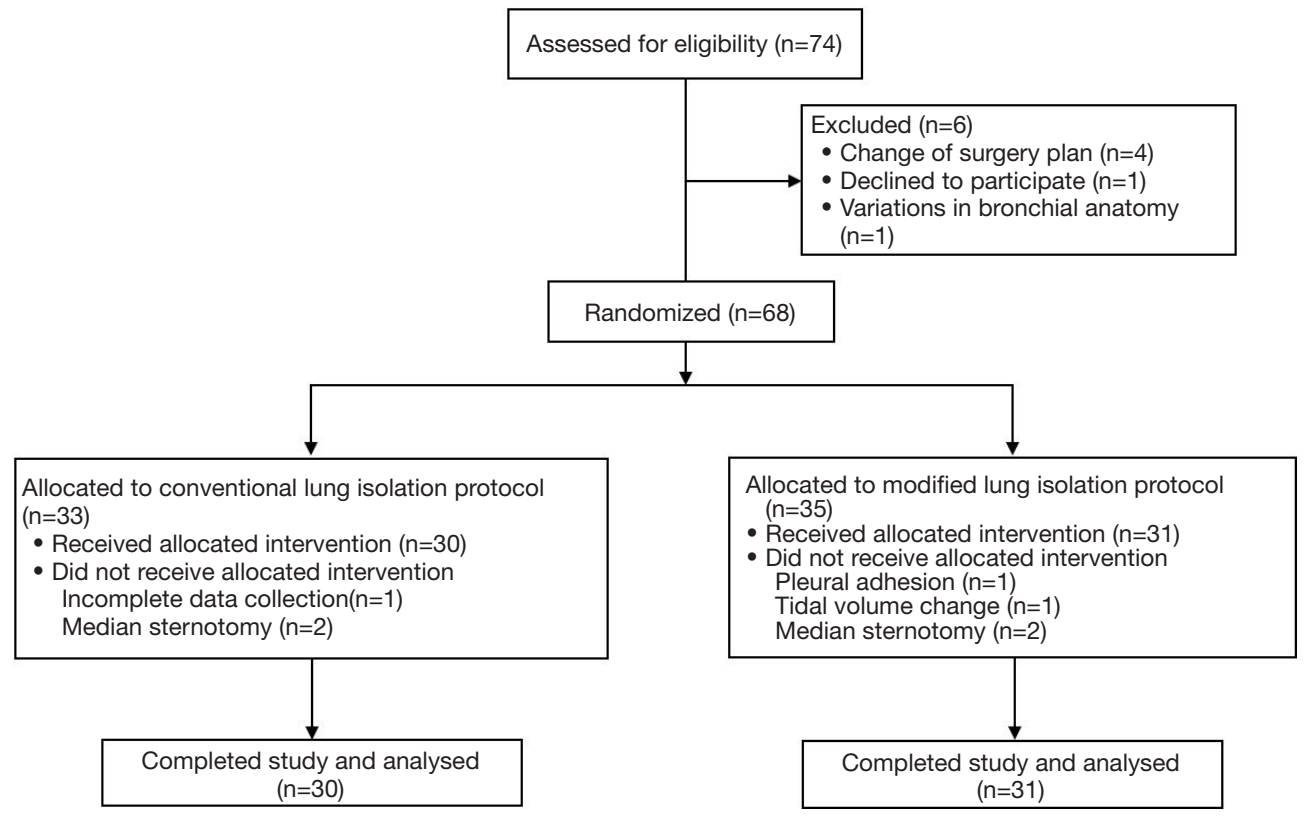

Figure 4 CONSORT diagram. CONSORT, consolidated standards of reporting trials.

and $\beta=0.2$. Considering a $20 \%$ loss, the number of selected patients in each group was 37 .

\section{Statistical analysis}

Statistical analysis was performed using SPSS version 25.0 software (IBM, Armonk, NY, USA). Data with normal distribution were expressed as mean \pm standard deviation, and those with non-Gaussian distribution were expressed as median and interquartile range. Discrete data were analyzed using the $\chi^{2}$-test or Fisher's exact test. Continuous data were compared using an independent sample $t$-test or Mann-Whitney $U$-test. $\mathrm{P}<0.05$ was considered statistically significant.

\section{Results}

A total of 74 patients were included in the present study. Of these, 6 were excluded and 68 were randomized; 33 cases were assigned to the group C, and 3 were excluded, and 35 cases were assigned to group $M$, and 4 cases were excluded (Figure 4). No statistical differences were observed for baseline characteristics, comorbidities, preoperative evaluations, and surgery types (Table 1).

The number of cases in which lung recruitment was performed due to hypoxemia during lung isolation after CPB in group C $(50 \%, 15 / 30)$ was significantly greater than in group $\mathrm{M}(16.1 \%, 5 / 31, \mathrm{P}=0.005)$. The number of cases requiring DLV in group C $(36.7 \%, 11 / 30)$ was notably greater when compared with group $\mathrm{M}(6.5 \%$, $2 / 31)(\mathrm{P}=0.004)$. The time for intubation and position confirmation in group $M$ was prominently longer than group C $\{390$ [344-480] s vs. 221 [179-278] s\}. Furthermore, dislodgements in both groups were similar (6vs. 7). There were 4 cases of airway bleeding in group $C$ and 3 in group $M$. Surgical field exposure was 5 [4.3-5] in group C, which was higher than that of group $M\{4$ [4-5], $\mathrm{P}=0.012\}$ (Table 2).

Five patients in group $M$ were changed to right lung isolation due to poor surgical field exposure. However, after excluding these cases, we also compared patients who successfully underwent SLB in group $M(n=26)$ with group C. The statistical significance of lung isolation-related parameters was the same as the previous result (Table 2). There were significant increases in the value of $\mathrm{P}_{\text {peak }}$ and $\mathrm{P}_{\text {plat }}$ during lung isolation in group $\mathrm{C}$, which were higher than that of group $M(n=26)$ before and after CPB $(P=0.002$ and $\mathrm{P}<0.001$ vs. $\mathrm{P}=0.008$ and $\mathrm{P}<0.001$, respectively) (Table 3).

Surgical hemostasis time was 47.5 [45-67] $\mathrm{min}$ in group $\mathrm{C}$ and 55 [47.5-63.5] $\mathrm{min}$ in group $\mathrm{M}(\mathrm{P}=0.294)$. Also, there was no significant difference in the following parameters: time durations of surgery, $\mathrm{CPB}$, and aortic clamping and in the volumes of fluid infusion, ultrafiltration, and urine output. The minimum hemoglobin level measured in group C was $103.7 \pm 16.3 \mathrm{~g} / \mathrm{L}$, which was not significantly 
Table 1 Demographic and patients characteristics

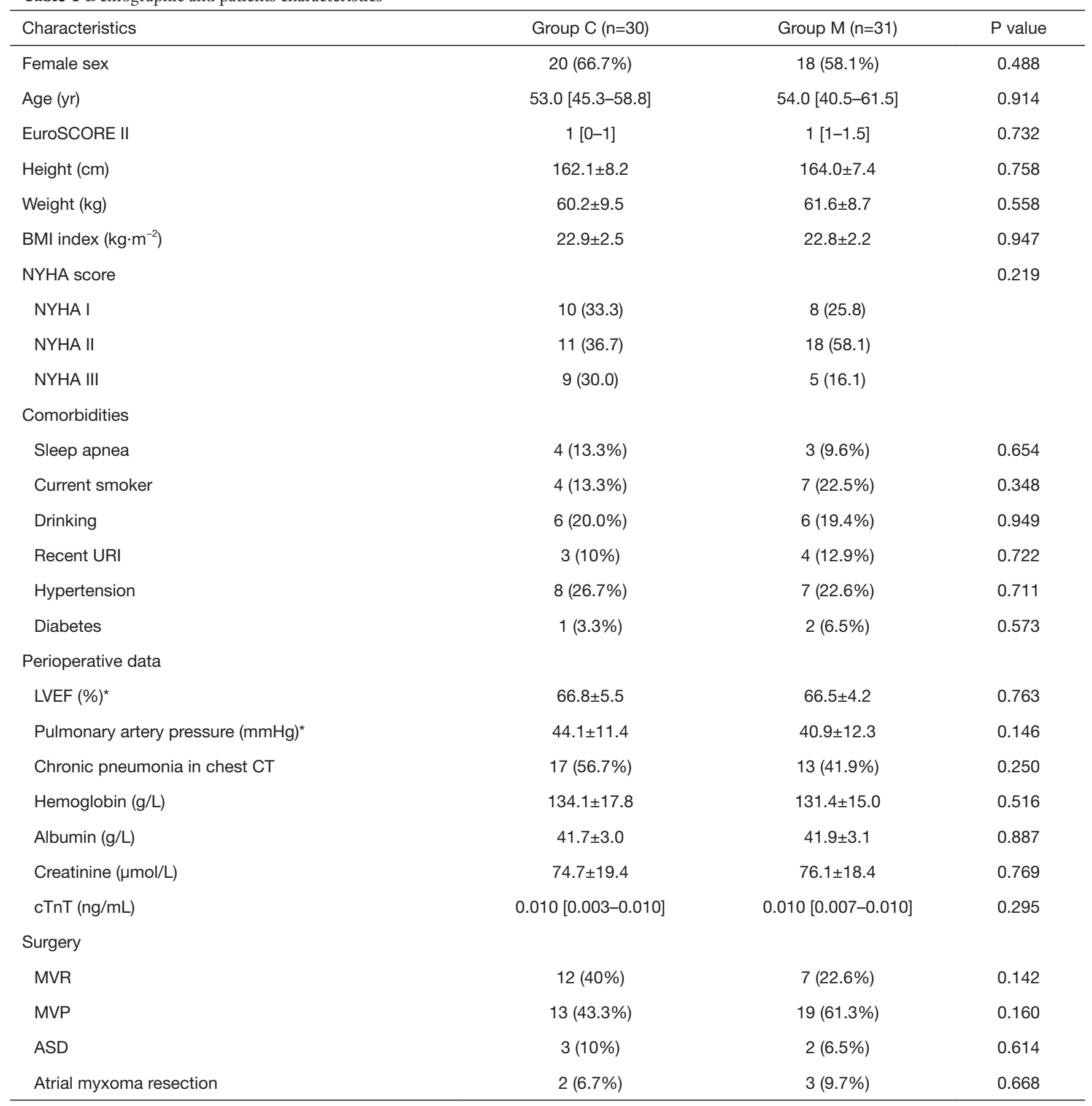

*, according to echocardiography. EuroSCORE, European system for cardiac operative risk evaluation; URI, upper respiratory infection; LVEF, left ventricular ejection fraction; cTnT, cardiac troponin T; MVR, mitral valve replacement; MVP, mitral valvuloplasty; ASD, atrial septal defect. 
Table 2 Lung isolation related parameters

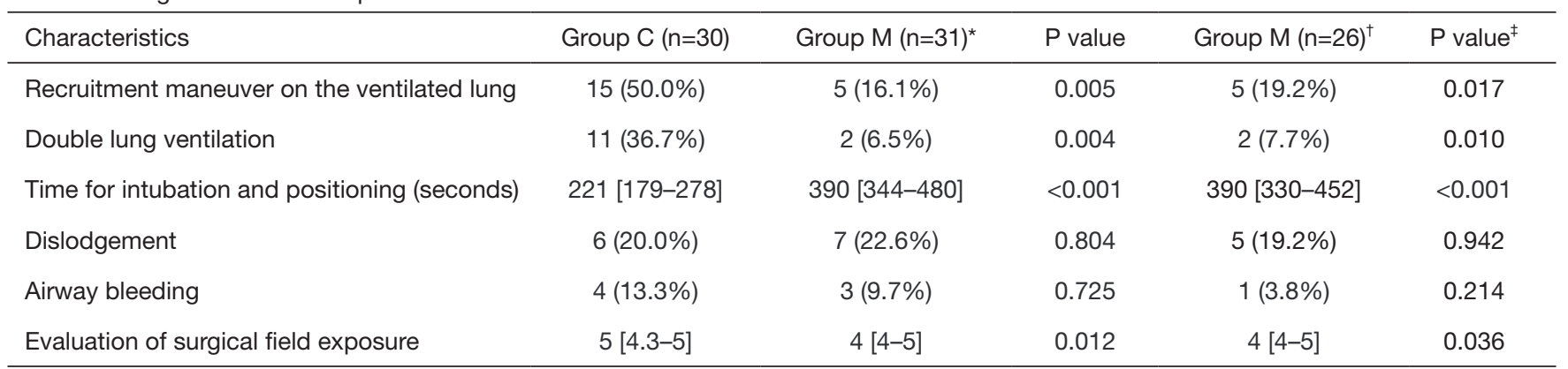

*, all cases in Group $M ;^{\dagger}$, cases in Group M with acceptable surgical field exposure; ${ }^{\ddagger}$, Group M (n=26) vs. Group C.

Table 3 Peak airway pressures $\left(\mathrm{P}_{\text {peak }}\right)$ and platform pressures $\left(\mathrm{P}_{\text {plat }}\right)\left(\mathrm{cmH}_{2} \mathrm{O}\right)$

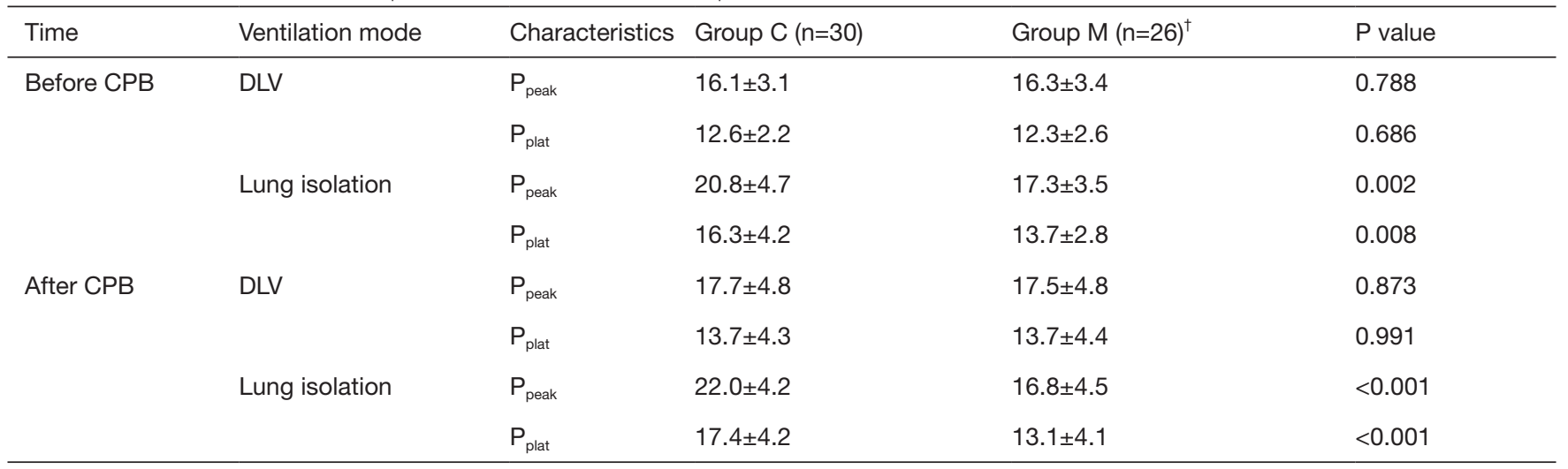

${ }^{\dagger}$, cases in Group M with acceptable surgical field exposure. CPB, cardiopulmonary bypass; DLV, double lung ventilation.

different from $103.9 \pm 12.6 \mathrm{~g} / \mathrm{L}$ in group $\mathrm{M}(\mathrm{P}=0.957)$. There was no perioperative allogeneic blood transfusion in either group. We found no significant differences in levels of postoperative maximum serum creatinine and cardiac troponin $\mathrm{T}$ and in the duration of postoperative mechanical ventilation, ICU discharge, and hospitalization between both groups (Table 4).

When comparing patients who successfully underwent SLB in group $M(n=26)$ with group $C$, no significant difference in $\mathrm{PaO}_{2} / \mathrm{FiO}_{2}$ at $\mathrm{T}_{0}, \mathrm{~T}_{2}$, and $\mathrm{T}_{3}(\mathrm{P}=0.605$, $\mathrm{P}=0.642$, and $\mathrm{P}=0.801$ ) was observed. $\mathrm{PaO}_{2} / \mathrm{FiO}_{2}$ at $\mathrm{T}_{1}$ in group $\mathrm{C}$ was $93.9 \pm 31.4 \mathrm{mmHg}$, which lower than group $\mathrm{M}$ (119.2 $\pm 52.3 \mathrm{mmHg}, \mathrm{P}=0.038) . \mathrm{PaO}_{2} / \mathrm{FiO}_{2}$ at $\mathrm{T}_{1}, \mathrm{~T}_{2}$, and $\mathrm{T}_{3}$ were inferior to $\mathrm{T}_{0}$ in both groups $(\mathrm{P}<0.01)$ (Table 5$)$.

\section{Discussion}

In the present study, we found that $\mathrm{PaO}_{2} / \mathrm{FiO}_{2}$ was markedly lower than the preoperative baseline after $\mathrm{CPB}$, even with DLV. Hypoxemia occurred in $50 \%$ of patients undergoing right lung isolation, while $36.7 \%$ required DLV to maintain oxygenation. Accordingly, satisfactory field exposure based on adequate oxygenation poses a challenge for anesthesiologists. The modified lung isolation strategy reduced the incidence of hypoxemia during lung isolation after CPB.

Although hypoxemia did not occur in all cases, the modified lung isolation strategy appeared to be more beneficial for patients with poor preoperative respiratory function and those at risk of hypoxemia during lung isolation after CPB. At the same time, SLB provides continuous lung isolation, which may be more appropriate for unskilled surgeons (9), as they can operate without the possibility of hypoxemia affecting the patient's safety during surgery.

BB was previously used for lung isolation in MICS (10), with only the right main bronchus blocked to achieve the right lung collapse. This might fail to treat possible hypoxemia after CPB, whereas SLB can notably increase the oxygenation index (11). In the case of robot-assisted 
Table 4 Intraoperative and postoperative data

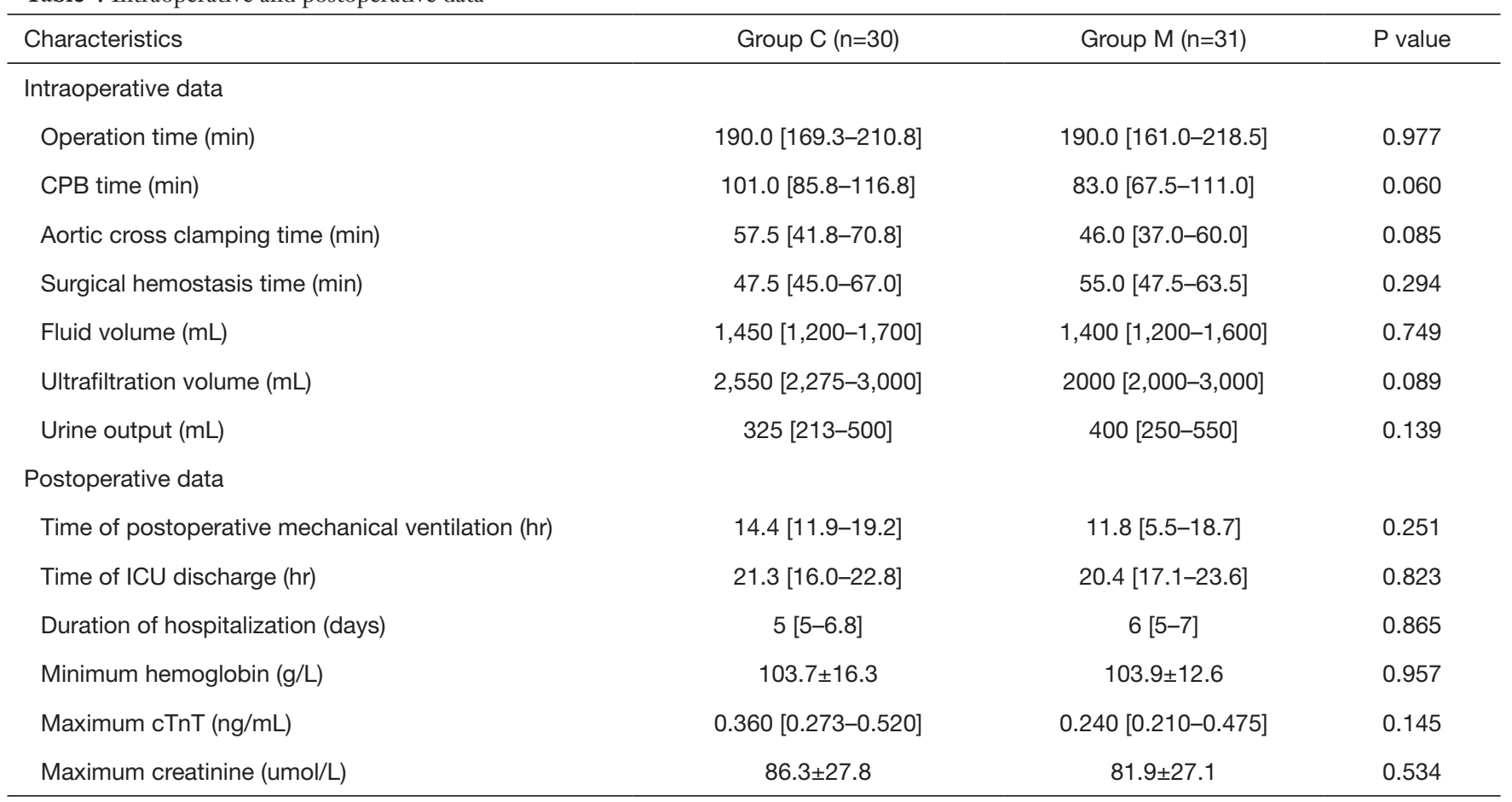

CPB, cardiopulmonary bypass.

Table $5 \mathrm{PaO}_{2} / \mathrm{FiO}_{2}(\mathrm{mmHg})$

\begin{tabular}{lccc}
\hline Time & $\begin{array}{c}\text { Conventional Group } \\
(\mathrm{n}=30)\end{array}$ & $\begin{array}{c}\text { Modified Group } \\
(\mathrm{n}=26)^{\dagger}\end{array}$ & $\mathrm{P}$ value \\
\hline $\mathrm{T}_{0}$ & $424.7 \pm 67.1$ & $416.2 \pm 52.9$ & 0.605 \\
$\mathrm{~T}_{1}$ & $93.9 \pm 31.4^{*}$ & $119.2 \pm 52.3^{*}$ & 0.038 \\
$\mathrm{~T}_{2}$ & $311.5 \pm 107.9^{*}$ & $325.6 \pm 117.3^{*}$ & 0.642 \\
$\mathrm{~T}_{3}$ & $359.7 \pm 105.3^{*}$ & $366.9 \pm 107.0^{*}$ & 0.801 \\
\hline${ }^{\dagger}$, cases in Group $\mathrm{M}$ with acceptable surgical field exposure. \\
$\mathrm{T}_{0}$ indicated data before anesthesia induction (FiO $\left.{ }_{2} 0.21\right) . \mathrm{T}_{1}$ \\
indicated data at 10 minutes after weaning from CPB (if DLV \\
was performed due to hypoxemia within 10 minutes, data would \\
be collected when the lowest $\mathrm{SpO}_{2}$ occurred in the next lung \\
isolation period). $\mathrm{T}_{2}$ indicated data after bilateral lung recruitment \\
at the end of surgery and $\mathrm{T}_{3}$ for 24 hours after surgery. ${ }^{*}$, vs. $\mathrm{T}_{0}$ \\
in each group, $\mathrm{P}<0.01$.
\end{tabular}

coronary artery bypass grafting, the patient can encounter severe hypoxemia when the $\mathrm{BB}$ is used to isolate the left lung. Subsequently, BB's cuff was placed forward into the left upper lobe bronchus for SLB, after which hypoxemia was resolved and it was possible to proceed with the operation (12). We first attempted to apply SLB in MICS through right lateral mini-thoracotomy with BB blocking the bronchus intermedius (Figure 5).

With the ventilated right upper lobe, $\mathrm{PaO}_{2} / \mathrm{FiO}_{2}$ during lung isolation after CPB was significantly higher in group $\mathrm{M}$ than group $\mathrm{C}$; however, it was quite low. Besides the impact of $\mathrm{CPB}$, it might be due to rare lung recruitment in group $\mathrm{M}, \mathrm{FiO}_{2}$ 1.0, and relatively low tidal volume during lung isolation without PEEP, all of which resulted in atelectasis and affected oxygenation (13). With the increased volume of the right upper lobe in group $\mathrm{M}, \mathrm{P}_{\text {peak }}$ and $\mathrm{P}_{\text {plat }}$ values in group $\mathrm{M}$ were remarkably lower than those in group $\mathrm{C}$ under the same tidal volume setting, reducing the collapsed lung volume and incidence of lung injury (14).

Field exposure was important for surgical hemostasis, along with no difference in postoperative minimum hemoglobin level and hemostasis time between the 2 groups in our study. The surgical field evaluation score was lower in group $M$ than group $C$. This could be due to surgeons feeling more comfortable operating in the cavity with the collapsed whole right lung and the original narrow vision becoming increasingly squeezed by the inflated right upper lobe, even when the operation targets were not covered. Also, for 5 cases in group $M$, the right upper lobe occluded the field at both inspiratory and expiratory phases, indicating that SLB might not be suitable for all patients. 

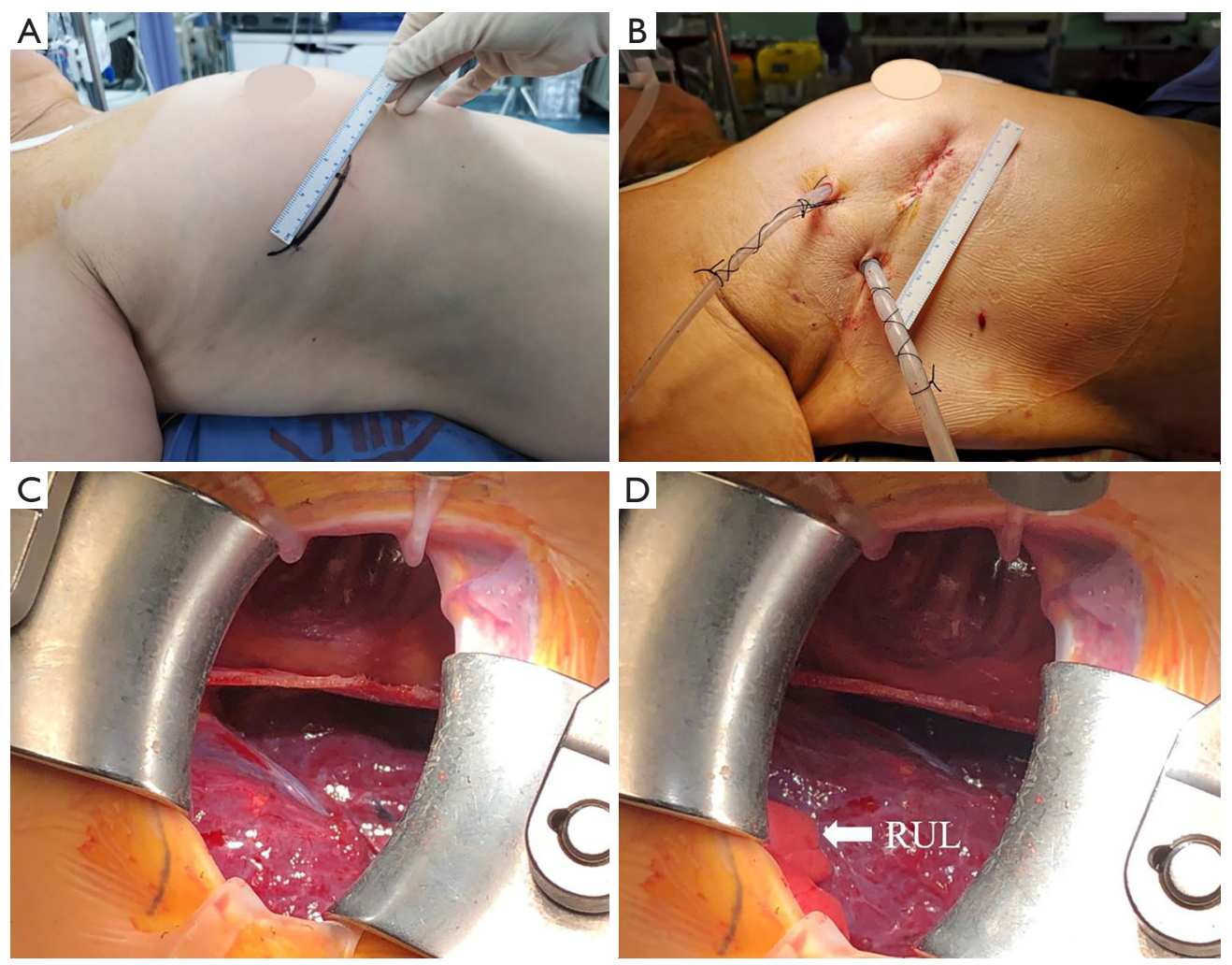

Figure 5 Patient position and surgical fields. (A) Patient position and incision mark of right lateral mini-thoracotomy; (B) Incision after surgery; (C) surgical field at the end of expiration, RUL was not showed; (D) surgical field at the end of inspiration, RUL was partly showed. RUL, right upper lobe.

However, the exposure significantly improved after the BB cuff was retracted to the right main bronchus.

The advantages of DLT for lung isolation include absolute lung separation, easy suction, convenient and reliable conversion between OLV, and DLV. The disadvantages include difficulty selecting the appropriate size, unsuitability for difficult airways, and possible tracheobronchial injury. BB's advantages include easy recognition of the carina anatomy through the trachea, suitability for difficult airway, and unnecessity to replace a tracheal tube after surgery. The disadvantages include difficult suction and potential dislodgement or loss of seal (15). It takes more time for BB to acquire a suitable position for lung isolation (16). Although lung isolation by the BB is a basic skill for anesthesiologists, the option may not be routine in clinical practice and requires a learning curve (17). When SLB is implemented, the time for positioning inevitably increases. The operation time is relatively shortened without replacing DLT with a singlelumen endotracheal tube after surgery. Airway edema can also occur after cardiac surgery, leading to the risk of replacing the endotracheal tube, where the $\mathrm{BB}$ has a prominent advantage (18). Also, we found no differences in airway bleeding and dislodgement, further supporting the modified lung isolation strategy's safety and efficacy.

The present study has some limitations. First, the surgeons were not blinded to the interventions. Furthermore, the evaluation for surgical field exposure was based on subjective feelings and lacked objective evidence for lung collapse (19). This could have biased the evaluation. Second, a previously published study showed no difference in the time taken to replace a DLT with a single-lumen endotracheal tube using an exchange catheter compared to the $\mathrm{BB}$, which was related to operation room efficiency (10). We did not collect related time data in our study, so lacking of the data to analyses the difference between two study groups. Finally, a recent study showed that the incidence of postoperative sore throat and hoarseness in patients using the $\mathrm{BB}$ were lower than DLT (20). In our study, patients in group C were reintubated with an accustomed single-lumen tracheal tube with a smaller 
size than that of group M (7.5\# for males, 7.0\# for females), so no relevant follow-up was performed.

\section{Conclusions}

The novel modified lung isolation strategy reduced hypoxemia during lung isolation after CPB in patients who underwent right lateral mini-thoracotomy, a MICS.

\section{Acknowledgments}

Funding: None.

\section{Footnote}

Reporting Checklist: The authors have completed the CONSORT reporting checklist. Available at http://dx.doi. org/10.21037/atm-20-986

Data Sharing Statement: Available at http://dx.doi. org/10.21037/atm-20-986

Conflicts of Interest: All authors have completed the ICMJE uniform disclosure form (available at http://dx.doi. org/10.21037/atm-20-986). The authors have no conflicts of interest to declare.

Ethics Statement: The authors are accountable for all aspects of the work in ensuring that questions related to the accuracy or integrity of any part of the work are appropriately investigated and resolved. The present study was conducted in accordance with the Declaration of Helsinki (as revised in 2013) and approved by the Ethics Committee of Zhongshan Hospital, Fudan University (approval number: B2016-022R). All patients provided signed informed consent.

Open Access Statement: This is an Open Access article distributed in accordance with the Creative Commons Attribution-NonCommercial-NoDerivs 4.0 International License (CC BY-NC-ND 4.0), which permits the noncommercial replication and distribution of the article with the strict proviso that no changes or edits are made and the original work is properly cited (including links to both the formal publication through the relevant DOI and the license). See: https://creativecommons.org/licenses/by-ncnd/4.0/.

\section{References}

1. Falk V, Cheng DCH, Martin J, et al. Minimally invasive versus open mitral valve surgery: a consensus statement of the international society of minimally invasive coronary surgery (ISMICS) 2010. Innovations 2011;6:66-76.

2. Downs EA, Johnston LE, LaPar DJ, et al. Minimally invasive mitral valve surgery provides excellent outcomes without increased cost: a multi-institutional analysis. Ann Thorac Surg 2016;102:14-21.

3. Schmitto JD, Mokashi SA, Cohn LH. Minimally-invasive valve surgery. J Am Coll Cardiol 2010;56:455-62.

4. Kottenberg-Assenmacher E, Kamler M, Peters J. Minimally invasive endoscopic port-access intracardiac surgery with one lung ventilation: impact on gas exchange and anaesthesia resources. Anaesthesia 2007;62:231-8.

5. Campos JH, Feider A. Hypoxia during one-lung ventilation-a review and update. J Cardiothorac Vasc Anesth 2018;32:2330-8.

6. Clark SC. Lung injury after cardiopulmonary bypass. Perfusion 2006;21:225-8.

7. Campos JH. Update on selective lobar blockade during pulmonary resections. Curr Opin Anaesthesiol 2009;22:18-22.

8. Ad N, Holmes SD, Massimiano PS, et al. The use of del Nido cardioplegia in adult cardiac surgery: A prospective randomized trial. J Thorac Cardiovasc Surg 2018;155:1011-8.

9. Sakaguchi T, Totsugawa T, Kuinose M, et al. Minimally invasive mitral valve repair through right minithoracotomy- 11-Year single institute experience. Circ J 2018;82:1705-11.

10. Grocott HP, Darrow TR, Whiteheart DL, et al. Lung isolation during port-access cardiac surgery: double-lumen endotracheal tube versus single-lumen endotracheal tube with a bronchial blocker. J Cardiothorac Vasc Anesth 2003;17:725-7.

11. Campos JH. Effects on oxygenation during selective lobar versus total lung collapse with or without continuous positive airway pressure. Anesth Analg 1997;85:583-6.

12. Agrawal DR, Nambala S, Fartado A. Selective lobar blockade in minimally invasive coronary artery bypass grafting: A technical advantage in patients with low respiratory reserve that precludes one-lung ventilation. Ann Card Anaesth 2016;19:542-4.

13. Minkovich L, Djaiani G, Katznelson R, et al. Effects of alveolar recruitment on arterial oxygenation in patients 
after cardiac surgery: a prospective, randomized, controlled clinical trial. J Cardiothorac Vasc Anesth 2007;21:375-8.

14. O'Gara B, Talmor D. Perioperative lung protective ventilation. BMJ 2018;362:k3030.

15. Campos JH. Which device should be considered the best for lung isolation: double-lumen endotracheal tube versus bronchial blockers. Curr Opin Anaesthesiol 2007;20:27-31.

16. Clayton-Smith A, Bennett K, Alston RP, et al. A comparison of the efficacy and adverse effects of doublelumen endobronchial tubes and bronchial blockers in thoracic surgery: a systematic review and meta-analysis of randomized controlled trials. J Cardiothorac Vasc Anesth 2015;29:955-66.

17. Rispoli M, Zani G, Bizzarri F, et al. Bronchial blocker positioning: learning curve and confidence in its use.

Cite this article as: Ren Y, Lyu Y, Yu Y, Jin L, Hu Y, Guo K, Cang J. Selective right middle and lower lobe blockade for minimally invasive cardiac surgery: a prospective, single-center, randomized controlled study. Ann Transl Med 2021;9(3):254. doi: 10.21037/atm-20-986
Minerva Anestesiol 2018;84:1254-60.

18. Vernick WJ, Woo JY. Anesthetic considerations during minimally invasive mitral valve surgery. Semin Cardiothorac Vasc Anesth 2012;16:11-24.

19. Bussieres JS, Somma J, Del Castillo JL, et al. Bronchial blocker versus left double-lumen endotracheal tube in video-assisted thoracoscopic surgery: a randomizedcontrolled trial examining time and quality of lung deflation. Can J Anaesth 2016;63:818-27.

20. Zhang C, Yue J, Li M, et al. Bronchial blocker versus double-lumen endobronchial tube in minimally invasive cardiac surgery. BMC Pulm Med 2019;19:207.

(English Language Editors: R. Scott and J. Chapnick) 